\title{
Design and Performance Enhancement of Vivaldi Antenna
}

Sunita Rani, Himanshu Monga, Silki Baghla

ECE Department, J.C.D.M College of Engineering, Sirsa, Haryana, India

J.C.D.M College of Engineering, Sirsa Haryana, India

Corresponding Author - Dr. Himanshu Monga

\begin{abstract}
In this paper, a computer aided design of Vivaldi Antenna is developed which is used to study the effect of different parameters such as rate of opening of exponential slot and size of the radius of circular slot of the Vivaldi Antenna. The proposed design resulted in enhancement of gain and reduction in reflection losses. COMSOL Multi physics simulator is used to design the proposed Vivaldi antenna.
\end{abstract}

Key words: Vivaldi antenna; COMSOL multiphysics; copper plate (FR4, 3.38 dielectric value)

Published Online: 31st Jan 2018

\section{Introduction}

The Vivaldi antenna is a special kind of tapered slot antenna (TSA), having an exponentially tapered slot profile. It is first recognized by Gibson in $1979^{[1]}$. It is useful for wide-band applications ${ }^{[4]}$. This research focused mainly on miniature antennas with a low losses and high gain. The aim of this research is to compute the far-field pattern and to compute the impedance matching of the structure.

The proposed Vivaldi antennas consist of a micro strip feed line, micro strip line to slot line transition, and the radiating structure. Radiating structure is exponential tapered and the radiation takes place along the axis of the tapering. ${ }^{[7-9]}$

This antenna comprises of three different types of slot lines which are:

(i) The circular slot which is used to realize the impedance matching of the micro strip

transmission line.

(ii) The rectangular slot which is used to couple the electromagnetic wave from the micro strip transmission line.

(iii) The exponential tapered slot which is used 


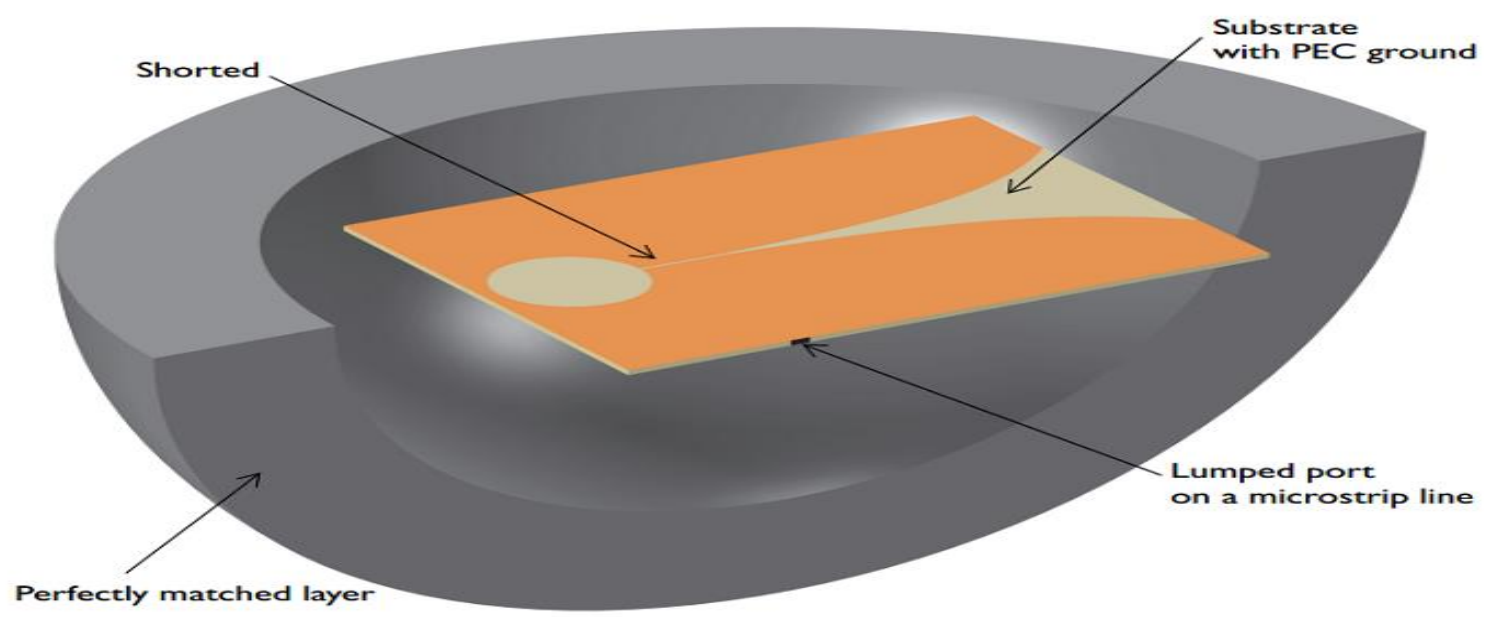

Figure 1. Vivaldi antenna structure

In this Vivaldi antenna model, the tapered slot is patterned with a prefect electric conductor (PEC) ground plane the top of the dielectric substrate .One end of the slot is open to the air and other end is finished with a circular slot. On the bottom of the substrate ,the shorted $50 \mathrm{Ohm}$ micro strip feed line is modeled PEC surface .The entire molding domain is bounded by perfectly matched layer (PEC) which act like an chamber absorbing all radiated energy .

\section{Related work}

Rajveer Dhawan [1] Presented the design and detailed results of the Vivaldi Antenna with the selection of FR4(2.33) substrate material. This antenna is showing remarkable performance at a frequency of $7.5 \mathrm{GHz}$ and VSWR equal to-24.5dB with high directivity and narrow beam width. Li Ying [3] proposed one design that starts from a single balanced Vivaldi antenna unit, designs and analyses the electromagnetic characteristics of balanced Vivaldi antenna array. Then get a high-gain more than $35 \mathrm{~dB}$ successfully by a parabolic antenna with the feed of $4 \times$ $3 \times 3$ Vivaldi array. ANU R G ${ }^{[8]}$ designed an antenna which used in the band from $2.1 \mathrm{GHz}$ to $9.2 \mathrm{GHz}$ with a fractional bandwidth of $125.66 \%$. Corresponding to the design frequency of $2.8 \mathrm{GHz}$ a peak gain of $6.122 \mathrm{~dB}$ and minimum $\mathrm{S} 11$ of $-28.5 \mathrm{~dB}$ is achieved.

\section{Design parameters of proposed antenna}

General parameters that are used during the design of Vivaldi are given in Table 1.1

\begin{tabular}{|l|l|l|l|}
\hline Name & Expression & Value & Description \\
\hline W-slot & $0.5[\mathrm{~mm}]$ & $5.000 \mathrm{E}-4 \mathrm{~m}$ & Slot width \\
\hline Thickness & $60[\mathrm{mil}]$ & $0.0015240 \mathrm{~m}$ & Substrate thickness \\
\hline F-max & $6.0[\mathrm{GHz}]$ & $6.0000 \mathrm{E} 9 \mathrm{~Hz}$ & $\begin{array}{l}\text { Maximum Frequency } \\
\text { in sweep }\end{array}$ \\
\hline F-min & $2.0[\mathrm{GHz}]$ & $2.0000 \mathrm{E} 9 \mathrm{~Hz}$ & $\begin{array}{l}\text { Minimum frequency } \\
\text { in sweep }\end{array}$ \\
\hline F0 & F-max & $6.5000 \mathrm{E} 9 \mathrm{~Hz}$ & $\begin{array}{l}\text { Current frequency in } \\
\text { sweep }\end{array}$ \\
\hline Length & $110[\mathrm{~mm}]$ & $011000 \mathrm{~m}$ & Length of antenna \\
\hline Radius & $10[\mathrm{~mm}]$ & $0.012000 \mathrm{~m}$ & Radius of circular slot \\
\hline Width & $80[\mathrm{~mm}]$ & $0.080000 \mathrm{~m}$ & Width of antenna \\
\hline
\end{tabular}




\begin{tabular}{|l|l|l|l|}
\hline Dielectric & 3.38 & 33800 & $\begin{array}{l}\text { Dielectric constant of } \\
\text { substrate }\end{array}$ \\
\hline
\end{tabular}

Table 1. Design parameters

\section{Design procedure}

The proposed VIVALDI antenna is designed using COMSOL simulate with following step:

i. Select the dimensions. ii. The Vivaldi antenna is realized on a thin dielectric substrate. The Vivaldi block with the dimensions given in Table 1.1 is drawn on substrate FR4. The length of the Vivaldi antenna is $110 \mathrm{~mm}$, thickness is $1.5 \mathrm{~mm}$ and breadth is $80 \mathrm{~mm}$.

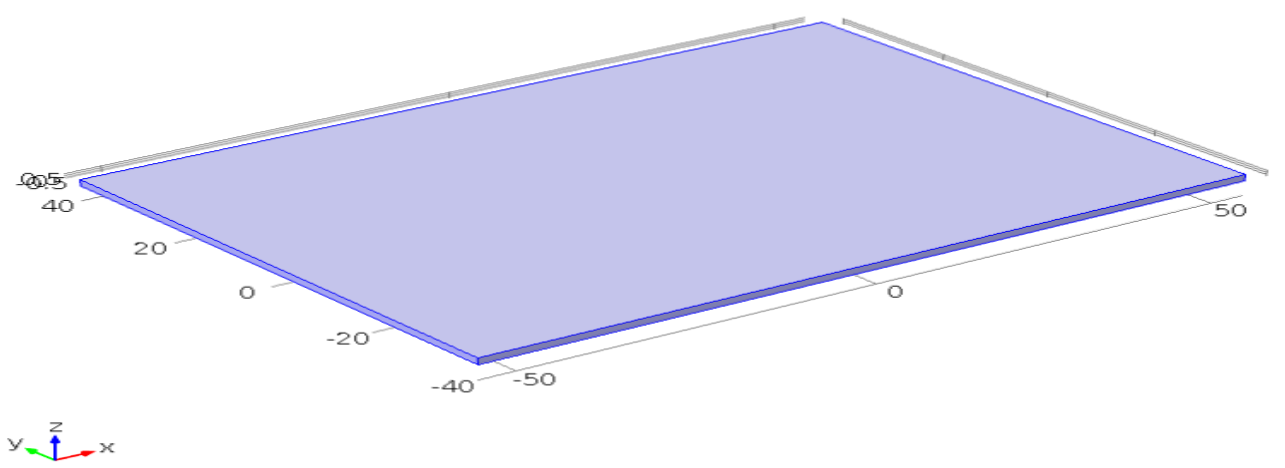

Figure 2. Substrate

iii. In the next step, feed line is selected. In this work. The feed line with the line width $=3.2$, Depth $=40+\mathrm{w} \_$slot $/ 2$, Height $=$ thickness breadth of $5 \mathrm{~mm}$ and length $16 \mathrm{~mm}$ is drawn. Shown in figure 3

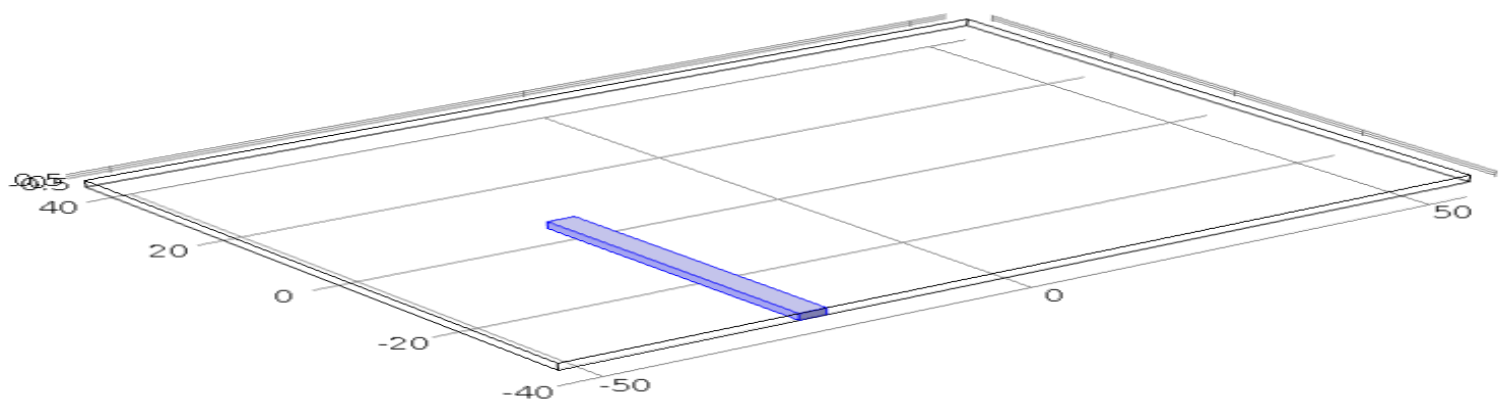

$y=t^{z}-x$

Figure 3. Feed line

iv. Under a Plane Geometry node we define and create the geometry sequence for a work plane. The Plane Geometry node also contains some settings for the visualization of the work plane's geometry. Plane geometry for the proposed antenna with the radius of $10 \mathrm{~mm}$ is show in fig 4 . 


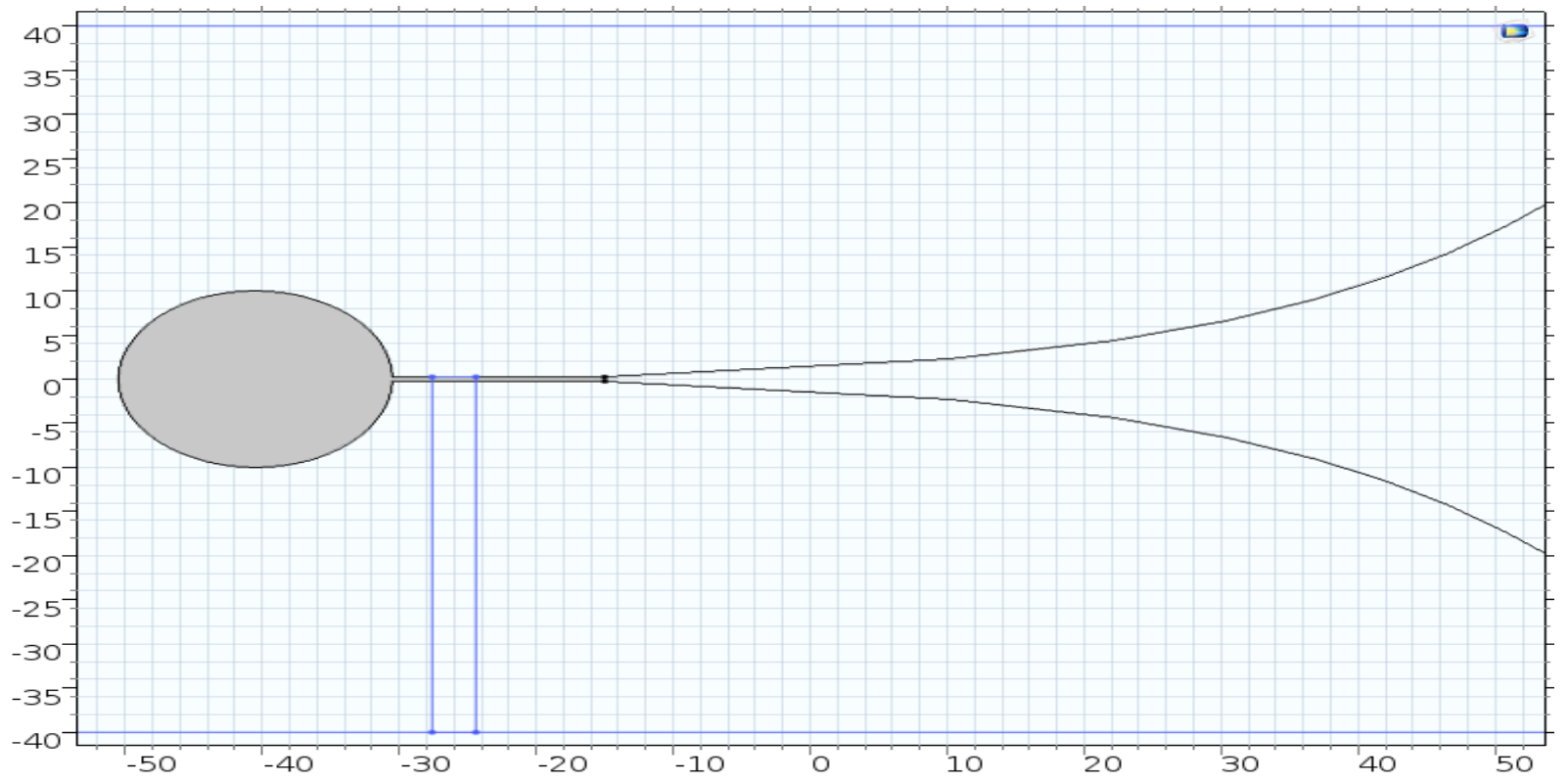

Figure 4. Plane geometry

v. Apply electromagnetic wave in frequency domain and the antenna is made up of FR4 material with electrical permeability.

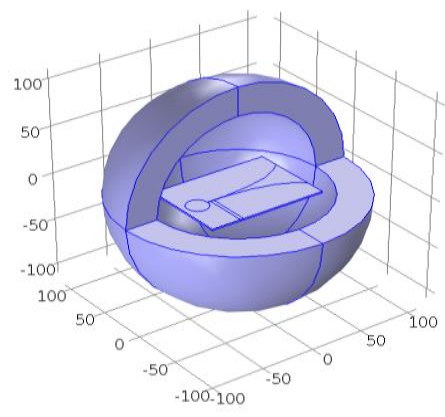

Figure 5. Electromagnetic waves, frequency domain

vi. In this step, the antenna will be divided into small part. A mesh imported into COMSOL can be used to construct geometry.

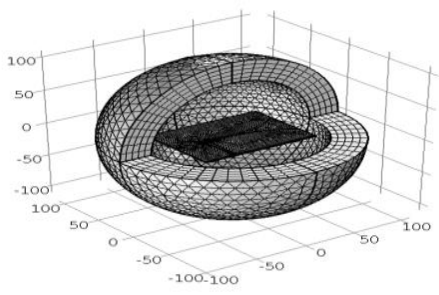

Figure 6. Mesh

\section{Discussion of results}

The proposed antenna is analyzed with variation in radius and it is found that at radius of $10 \mathrm{~mm}$. The designed antenna provide maximum electric field of 64 as show in fig.7. When then electric field of Vivaldi antenna is 64 the designed antenna promotes the gain from $7.95 \mathrm{~dB}$ in the desired frequency band (2 to $6.5 \mathrm{GHz}$ ) as shown in fig 8 . The improved antenna gain can be used for applications like microwave imaging and radars. The result of our simulation can be seen in the form of SWR plot. According to our result SWR parameter is minimum 1.14 at $3.5 \mathrm{GHz}$ as shown in fig 9. Figure10 Show the Far field radiation pattern for FR4, beginning frequency is $2 \mathrm{GHz}$ and $6.5 \mathrm{GHz}$ that is the finish frequency. In this far field radiation pattern diagram highest output frequency at $4.5 \mathrm{GHz}$. With the selection of feed purpose employed in this model, the $\mathrm{S}_{11}$ parameter is minimum at $3.5 \mathrm{GHz}$ as shown in fig. 11.Figure12 shows a good Impedance matching occur at $3.5 \mathrm{GHz}$. 


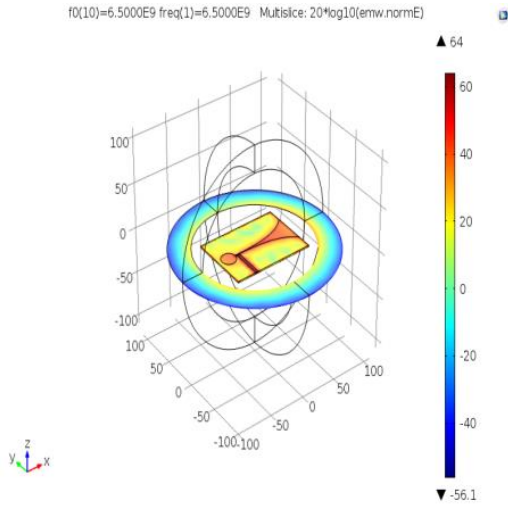

Figure7. Electric field of Vivaldi antenna

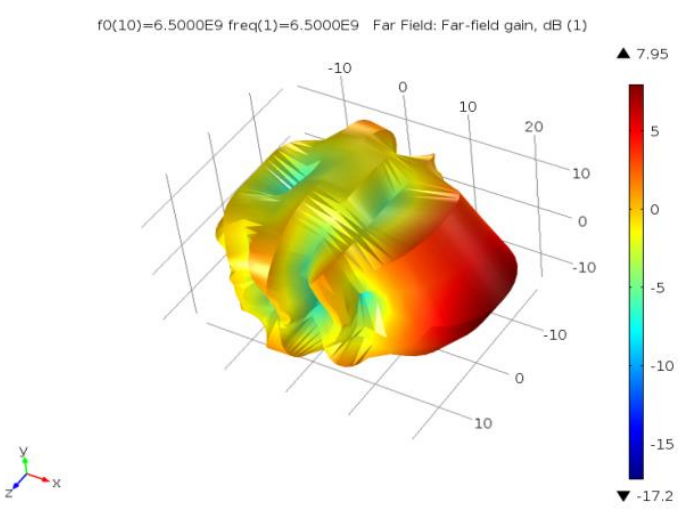

Figure 8. Gain of Vivaldi antenna

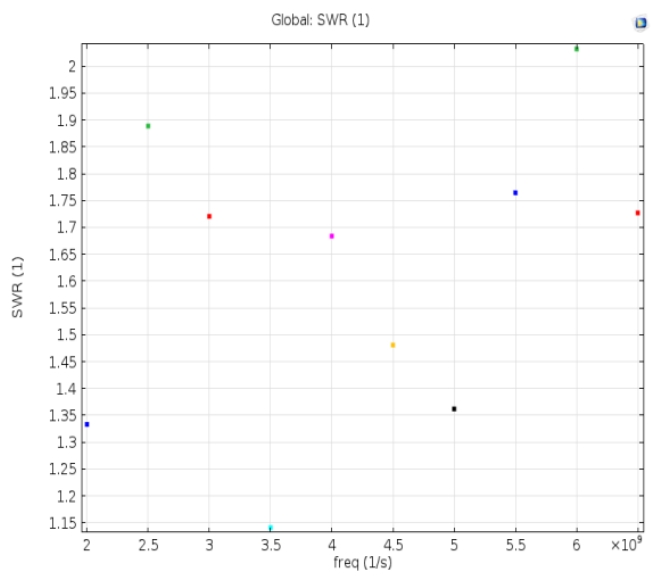

Figure 9. (SWR)

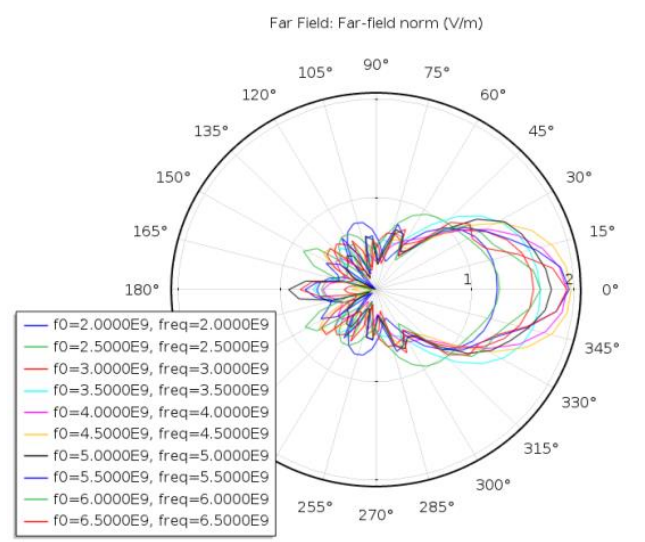

Figure 10. Far field radiation pattern

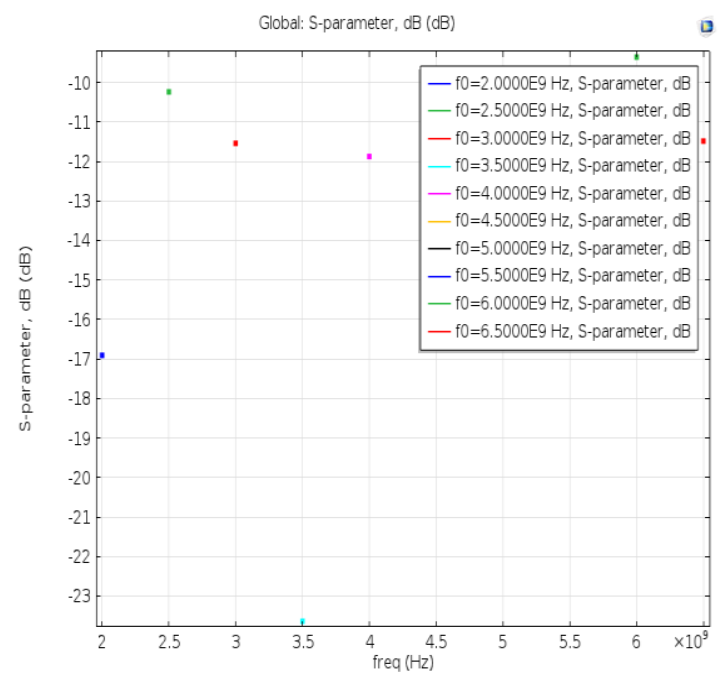

Figure 11. S-parameter

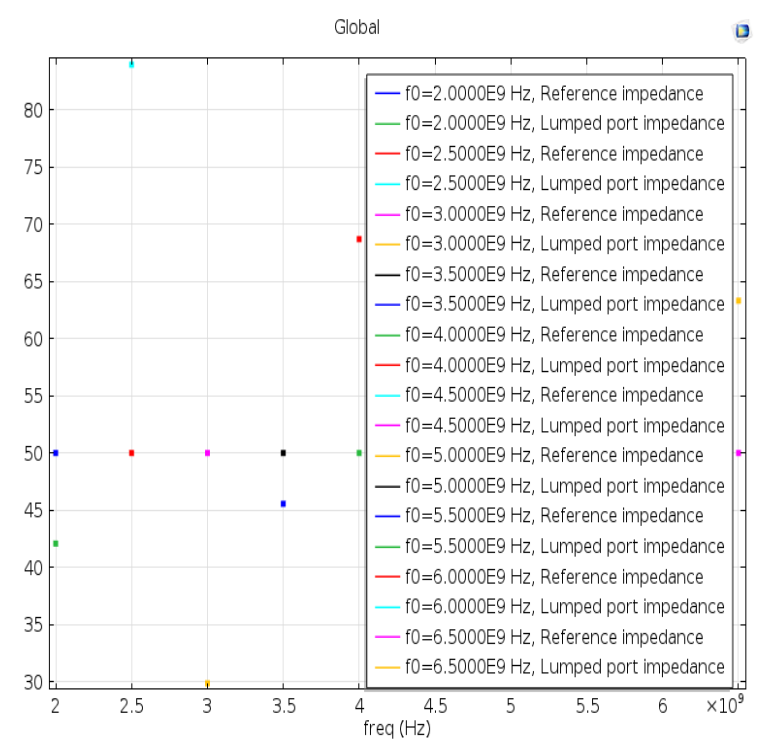

Figure 12. 1D impedance matching

\section{Conclusion}

The proposed Vivaldi antenna is simulated using the COMSOL. The proposed antenna is low- profile and uses low cost FR4 substrate. Here the geometry is not restricted and it take a shape such that the objective of maximum gain and minimum S11 is achieved. The designed antenna can be design frequency from 2 to 6.5 GHz. This antenna is showing good impedance matching performance at a frequency of $3.5 \mathrm{GHz}$. The proposed antenna is giving high electric field of 64 . low reflection losses are -24 and gain of antenna is $7.95 \mathrm{db}$. The proposed antenna is low-profile and uses low-cost FR-4 substrate. To validate the simulation results, the antenna would be fabricated and tested in near future.

\section{References}

[1] Rajveer Dhawan And Gurkiran Deep Kaur, "Vivaldi Antenna Simulation On Defining Parameters, 
Parametric Study And Results" ,I J C T A, Pp. 5129-5138 @ International Science Press, 9(11) 2016

[2] Matthias John, Max J. Ammann And Patrick Mcevoy "Uwb Vivaldi Antenna Based On A Spline Geometry With Frequency Band-Notch ", Ieee, 978-1-4244-2042-1/08/\$25.00 C IEEE, 2008

[3] Y. Yang, Y. Wang, And A. E. Fathy,"Design Of Compact Vivaldi Antenna Arrays For UWB See Through Wall Applications" Progress In Electromagnetics Research, Pier 82, 401-418, 2008

[4] Xu Han Lei Juan Cui Changjuan Ang Lin, "Uwb Dual Polarized Vivaldi Antenna With High Gain" 978-1-4673-2185-3/12/\$31.00 IEEE,2012

[5] D. Ziani Kerarti, F.Z Marouf , S.M. Meriah "New Tapered Slot Vivaldi Antenna For UWB Applications" 24th International Conference On Microelectronics (Icm), 2012

[6] Debdeep Sarkar, Kumar Vaibhav Srivastava "Srr-Loaded Antipodal Vivaldi Antenna For Uwb Applications With Tunable Notch Function" Proceedings Of The International Symposium On Electromagnetic Theory, 2013.

[7] Chittajit Sarkar "'Some Parametric Studies On Vivaldi Antenna International Jornal Of U -And
-E Service Science And Technology Vol.7 No 4,Pp 323 328, 2014.

[8] Anu R G S.Santhosh Kumar," Tapered Vivaldi Antenna Design Usingfourier Series Approach For Uwb Applications" 2014 First International Conference On Computational Systems And Communications (Iccsc) | 17-18 December 2014. [9] G. K. Pandey, H. S. Singh, P. K. Bharti, A. Pandey, And M. K. Meshram " High Gain Vivaldi Antenna For Radar And Microwave Imaging Applications " International Journal Of Signal Processing Systems Vol. 3, No. 1, June 2015.

[10] Kalyani P. Dakhale, 2m .S. Narlawar "Design Of Novel Vivaldi Antenna For Cognitive Radio Application In Ultra Wide Band" Volume-3, Issue-3, March-2015.

[11] Osama M. Dardeer, Tamer G. Abouelnaga, Ashraf S. Mohra, Hadia M. El-Hennawy "A Novel Uwb Vivaldi Antenna Array For Radar Applications" International Journal Of Scientific \& Engineering Research, Volume 7, Issue 5, May-2016.

[12] Li Ying, Chen Ai-Xin" Design And Application Of Vivaldi Antenna Array" 978-1-4244-2193-0/08/\$25.00 @ IEEE, 2008. 